\title{
PROCESSO DE OSMOSE DIRETA PARA A GERAÇÃO DE ENERGIA POR GRADIENTE DE SALINIDE / INVESTIGAÇÃO DE UM PROTÓTIPO DE FO/PRO UTILIZANDO MÓDULOS DE FIBRAS OCAS
}

\author{
N. J-D. R. MERMIER ${ }^{1}$, C. PIACSEK BORGES ${ }^{2}$ \\ ${ }^{1}$ Programa de Engenharia Química/COPPE, Universidade Federal do Rio de Janeiro, Cidade \\ Universitária, CP 68502, Rio de Janeiro, 21945-970, RJ, Brasil \\ E-mail para contato: nicolas@ @eq.coppe.ufrj.br
}

\begin{abstract}
RESUMO - O fenômeno de osmose é conhecido há mais de 150 anos e envolve o contato de uma membrana semipermeável com meios de diferentes tonicidades. A utilização do fluxo osmótico permeado através da membrana possibilita o acionamento de geradores de energia elétrica que pode ser utilizado como fonte renovável e sem impacto ambiental. Este trabalho consiste na montagem de um protótipo de FO/PRO para a geração de energia por gradiente de salinidade. As membranas produzidas são do tipo fibras ocas de acetato de celulose. O protótipo permite o acoplamento de 3 permeadores conectados em paralelo sendo a área efetiva de membrana por módulo de aproximadamente $2 \mathrm{~m}^{2}$. O fluxo permeado $\left(3,5 \mathrm{~L} / \mathrm{m}^{2} \cdot \mathrm{h}, \mathrm{MgSO}_{4}\right.$ 0,03M, $\pi$ : 8bar) obtido com as membranas produzidas nesse trabalho é comparável com a das membranas comerciais.
\end{abstract}

\section{INTRODUÇÃO}

A necessidade de diminuir a dependência mundial do consumo de combustível fóssil é reconhecida em praticamente todos os seguimentos da sociedade. Fontes de energia menos poluentes e renováveis possibilitam controlar e diminuir a emissão de gases nocivos ao meio ambiente, promovendo maior sustentabilidade. Ainda pouco explorada, a utilização do fluxo osmótico permeado através de uma membrana semipermeável possibilita o acionamento de geradores de energia elétrica que pode ser utilizado como fonte renovável e sem impacto ambiental. Esta fonte possui um grande potencial no Brasil, visto seu extenso litoral que permite o encontro da água doce com a água salgada. Estimativas indicam que no mundo, a energia disponível através de gradientes de salinidade é da ordem de 2.000 TWh por ano (Aaberg, 2003).

Embora este conceito seja bastante antigo, o processo ainda está enfrentando algumas dificuldades para ser amplamente operacionalizado por falta de membranas adequadas que devem tornar o processo economicamente mais competitivo. Atualmente, uma das poucas iniciativas no desenvolvimento de escala piloto e planta de processo de geração de energia elétrica por osmose direta é feita pela empresa norueguesa STATKRAFT. No Brasil, o laboratório de processos de separação com membranas da COPPE/UFRJ vem envolvido-se nas pesquisas de síntese de 
membranas e processos de separação durante quase três décadas, sendo uma referência nacional e internacional no campo.

Este trabalho consiste na produção de membranas poliméricas anisotrópicas do tipo fibras ocas integrais de acetato de celulose que permitam ampliar o fluxo permeado, mantendo sua restrição à passagem de sais dissolvidos. Estas fibras são acondicionadas em permeadores que possibilitam a passagem das soluções de diferentes salinidades em lados distintos da membrana. O protótipo permite o acoplamento de 3 módulos conectados em paralelo sendo a área efetiva de membrana por módulo de aproximadamente $2 \mathrm{~m}^{2}$ e de uma densidade de empacotamento de 2000 $\mathrm{m}^{2} / \mathrm{m}^{3}$. A construção destes permeadores também é objeto de estudo e inovação, principalmente, através da otimização da transferência de massa decorrente do controle da velocidade de escoamento dos fluidos dentro dos módulos.

\section{METODOLOGIA}

Membranas poliméricas anisotrópicas do tipo fibras ocas foram produzidas por inversão de fase em banho de não solvente. O polímero empregado é o acetato de celulose (AC) de massa molar numérica média de $50.000 \mathrm{~g} / \mathrm{mol}$. Os solventes empregados são a acetona $(\mathrm{AO}, 99,5 \%)$ e o formamide (FO, 99,5\%). A composição da solução polimérica empregada é AC/AO/FO (25/45/30 $\% \mathrm{~m} / \mathrm{m})$.

\subsection{PROCESSO DE FIAÇÃO}

A Figura 1 apresenta o equipamento de fiação por extrusão simples. Neste equipamento, a solução polimérica é bombeada em direção a extrusora por pressurização do tanque de alimentação com um gás inerte $\left(\mathrm{N}_{2}\right)$. Um controlador de velocidade permite ajustar a vazão do líquido interno por intermédio de uma bomba de engrenagem.

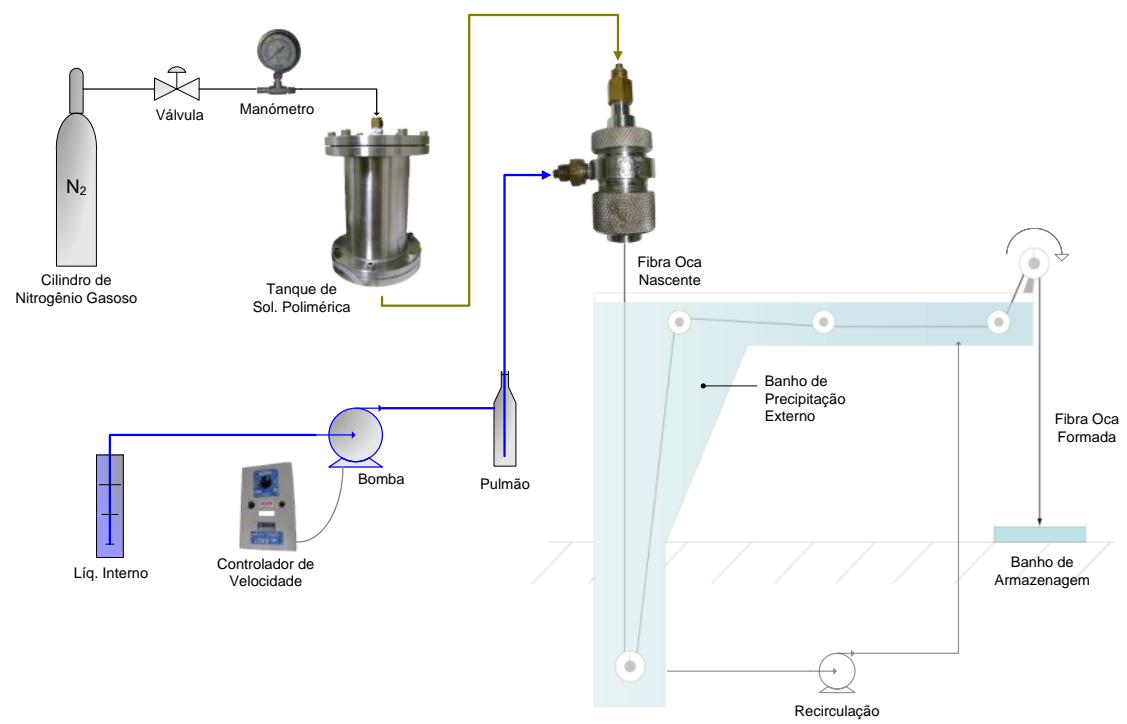

Figura 1 - Processo de fiação, no caso específico da extrusão simples. 


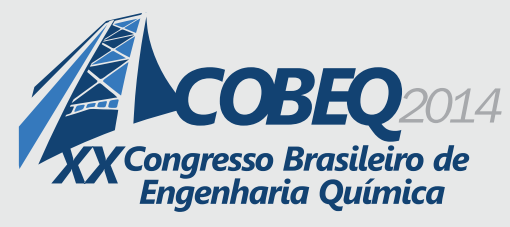

19 a 22 de outubro de 2014

Florianópolis/SC

As condições experimentais referentes à síntese das membranas do tipo fibras ocas por inversão de fase são apresentadas na Tabela 1.

Tabela 1 - Condições de fiação.

\begin{tabular}{|c|c|c|c|c|c|c|c|c|c|}
\hline $\begin{array}{l}\text { Fibras } \\
\text { Ocas } \\
\text { Ref. }\end{array}$ & $\begin{array}{l}\text { DEB } \\
{[\mathrm{cm}]}\end{array}$ & $\begin{array}{c}\text { Sol. } \\
\text { Polimérica } \\
\text { Vazão } \\
\text { Mássica } \\
\text { [g/min] }\end{array}$ & $\begin{array}{c}\text { Líq. Interno } \\
\text { Composição } \\
{[-]}\end{array}$ & $\begin{array}{c}\text { Líq. } \\
\text { Interno } \\
\text { Vazão Vol. } \\
\text { [ml/min] }\end{array}$ & $\begin{array}{c}\text { Velocidade } \\
\text { de Fiação } \\
\text { [m/min] }\end{array}$ & $\begin{array}{c}\text { Tempo de } \\
\text { Exp. ao } \\
\text { Ambiente } \\
\text { [s] }\end{array}$ & $\begin{array}{c}\text { Diâmetro } \\
\text { Externo da } \\
\text { Fibra }[\mu \mathrm{m}]\end{array}$ & $\begin{array}{c}\text { Diâmetro } \\
\text { Interno da } \\
\text { Fibra }[\mu \mathrm{m}]\end{array}$ & $\begin{array}{c}\text { Largura da } \\
\text { Parede da } \\
\text { Fibra }[\mu \mathrm{m}]\end{array}$ \\
\hline AC_2B & 3 & 2 & $\begin{array}{c}\mathrm{H}_{2} \mathrm{O} / \mathrm{NMP} \\
(10: 90)\end{array}$ & 0,7 & {$[4-5]$} & {$[0,3-0,5]$} & 700 & 400 & 150 \\
\hline
\end{tabular}

Caracterização das membranas produzidas: As membranas sintetizadas foram caracterizadas por microscopia eletrônica de varredura (MEV). A Figura 2 apresenta as fotomicrográficas de uma fibra decorrente da batelada AC_2B. A membrana apresenta uma estrutura anisotrópica, densa na região próxima à superfície externa, considerada como a camada seletiva, suportada por uma estrutura microporosa com poros interconectados. Observe-se que a morfologia do suporte é isenta de macrovazio, o que lhe confere uma resistência mecânica adaptada a pressão hidrostática de trabalho. A pressão limite superior tolerada pelas membranas produzidas é aproximadamente de 6bar.

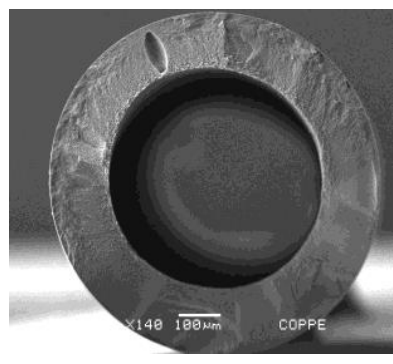

(a)

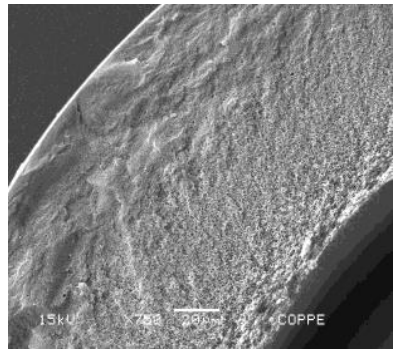

(d)

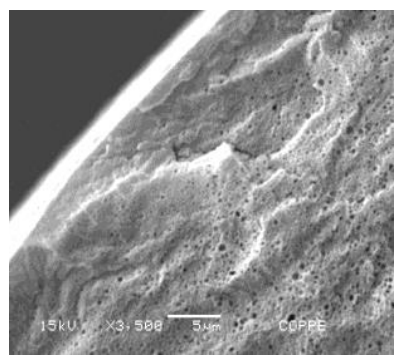

(b)

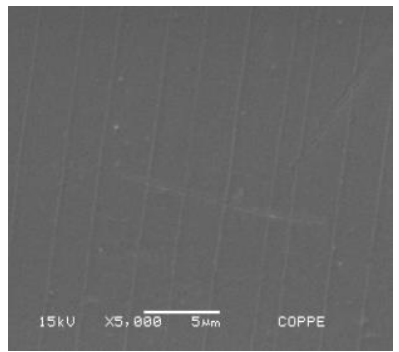

(e)

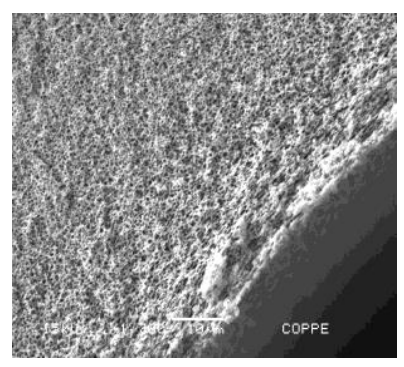

(c)

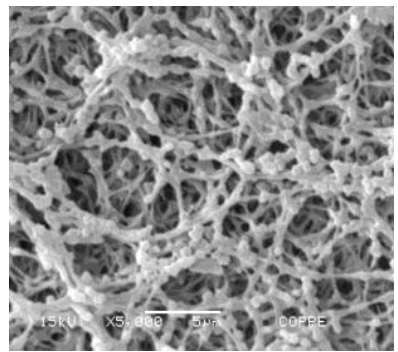

(f)

Figura 2 - Fotomicrográficas das diferentes regiões da fibra AC_2B: (a) a seção transversal; (d) a seção transversal detalhada; (b) a região próxima à superfície externa; (c) a região próxima à superfície interna; (e) a superfície externa; (f) a superfície interna. 


\section{PROTÓTIPO}

A Figura 3 representa o fluxograma do protótipo de geração de energia por osmose direta.

(a)

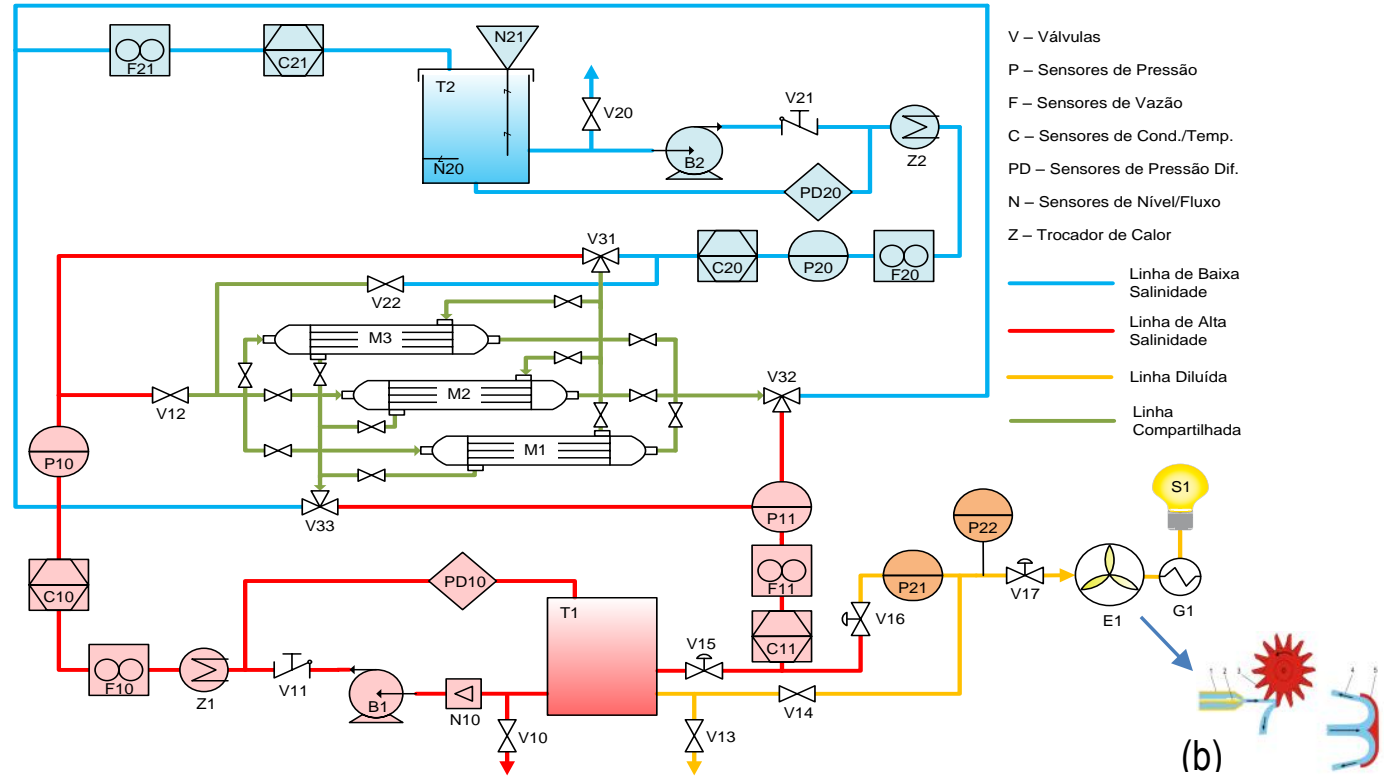

Figura 3 - (a) Fluxograma do protótipo de geração de energia; (b) Representação esquemática do funcionamento da turbina Pelton.

O sistema é baseado em uma corrente de alta salinidade e uma corrente de baixa salinidade circulando pelos permeadores. O fluxo de água permeado por osmose direta aumenta a vazão da corrente de alta salinidade que está em circuito fechado pressurizado. A linha diluída do fluxograma corresponde a corrente originada pelo excesso de água circulando. Essa variação de volume pode ser então reaprovada e direcionada para a turbina Pelton. A figura 4 apresenta uma visão global do protótipo e destaca a turbina que converte a energia hidrostática da água em energia cinética.

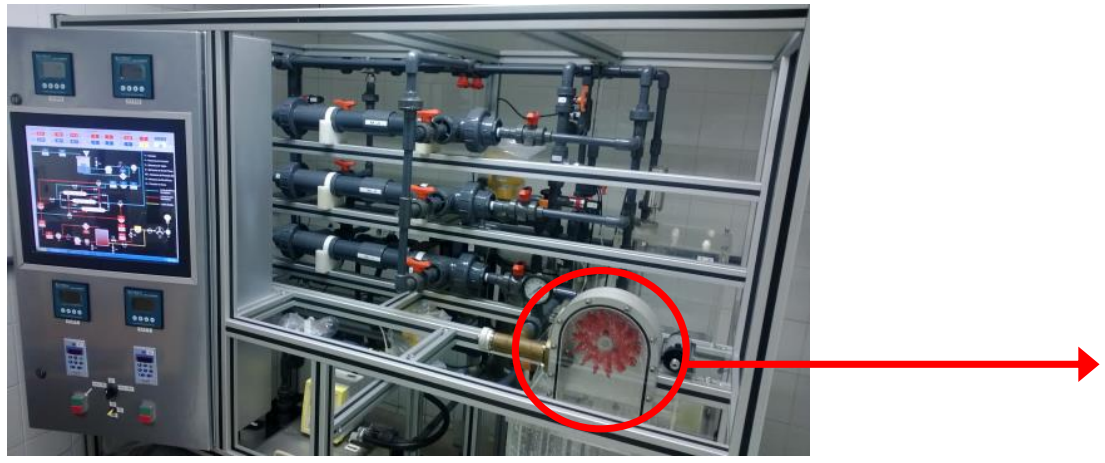

(a)

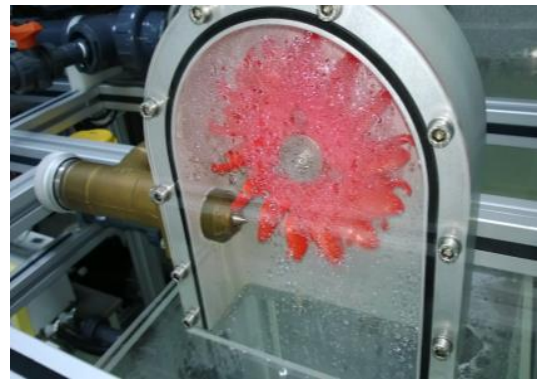

(b)

Figura 4 - (a) Protótipo de geração de energia por osmose direta; (b) Turbina Pelton 


\section{PERMEADORES}

O protótipo consiste em 3 módulos montados em paralelo. A alimentação ocorre de forma axial, pelo lúmen das fibras, e de forma lateral, na casca das fibras. As duas correntes circulam em contracorrente dentro dos permeadores o que favorece a transferência de massa. A versatilidade do sistema permite inverter as diferentes linhas no caso de membranas apresentando outras morfologias. A figura 5 apresenta a configuração dos permeadores e os diferentes sentidos das correntes ao longo das fibras ocas.

(a)
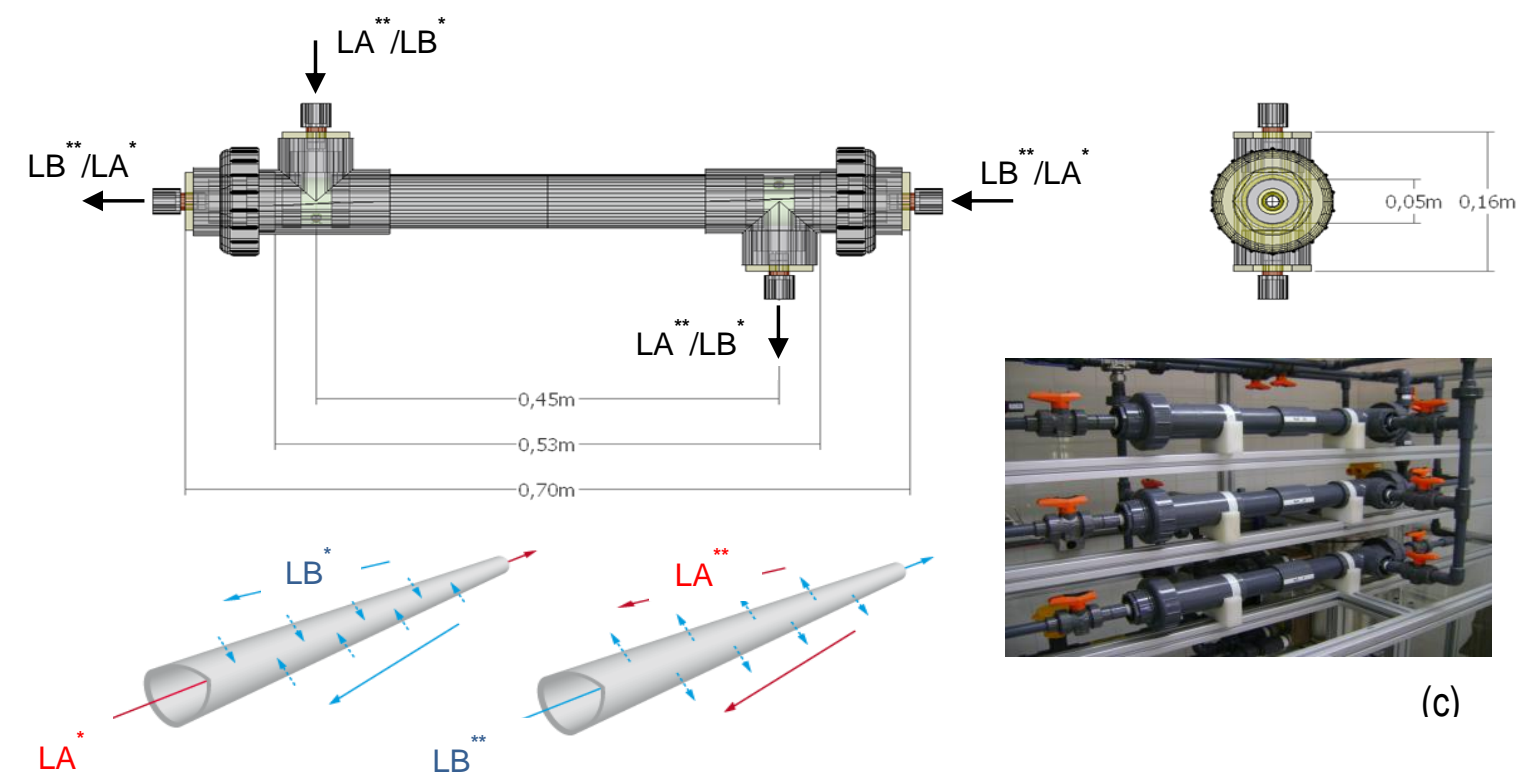

(b)

Figura 5 - (a) Representação esquemática e dimensionamento de um permeador; (b)

Direções possíveis das correntes de alta salinidade (LA), baixa salinidade (LB) e do fluxo osmótico permeado através das membranas; (c) Módulos de osmose direta montados em paralelo no protótipo.

\subsection{CARACTERIZAÇÃO DOS PERMEADORES / RESULTADOS}

Dois permeadores (M1, M2) montados em paralelo apresentando uma área especifica total de aproximadamente $1,5 \mathrm{~m}^{2}$ foram caracterizados afim de determinar as propriedades de transporte das membranas e seu desempenho em relação a geração de energia.

Teste de osmose inversa: Nesse teste, os permeadores foram caracterizados quanto à permeabilidade hidráulica e a rejeição ao sulfato de magnésio $\left(\mathrm{MgSO}_{4} 0,05 \mathrm{M}\right)$ através um teste de osmose inversa. 
A tabela 2 apresentam os resultados referentes à permeabilidade hidráulica e a rejeição ao sulfato de magnésio $(0,05 \mathrm{M})$.

Tabela 2 - Propriedades de transporte dos permeadores

\begin{tabular}{|c|c|c|c|c|c|}
\hline $\begin{array}{c}\text { Permeadores } \\
\text { Ref. }\end{array}$ & $\begin{array}{c}\mathrm{n}^{\circ} \text { Fibras úteis } \\
{[-]}\end{array}$ & $\begin{array}{c}\text { Área útil de } \\
\text { permeação } \\
{\left[\mathrm{m}^{2}\right]}\end{array}$ & $\begin{array}{c}\text { Resistência } \\
\text { hidráulica máxima } \\
{[\text { bar] }}\end{array}$ & $\begin{array}{c}\text { Permeabilidade } \\
\text { hidráulica }(\mathrm{A}) \\
{\left[\mathrm{L} / \mathrm{m}^{2} \cdot \mathrm{h} \cdot \mathrm{bar}\right]}\end{array}$ & $\begin{array}{c}\text { Rejeição [\%] } \\
\left(\mathrm{MgSO}_{4} 0,05 \mathrm{M}\right)\end{array}$ \\
\hline $\mathrm{M} 1+\mathrm{M} 2$ & 1300 & 1,5 & 5 & 1 & 72 \\
\hline
\end{tabular}

Teste de osmose direta: 0 desempenho dos permeadores foi avaliado pelo teste de osmose direta. As condições de operação são resumidas na tabela 3.

Tabela 3 - Condições de operação.

\begin{tabular}{|c|c|c|c|c|c|c|c|}
\hline \multirow[b]{2}{*}{ Correntes } & \multirow[b]{2}{*}{$\begin{array}{l}\text { Composição } \\
\text { (M) }\end{array}$} & \multirow[b]{2}{*}{$\begin{array}{l}\text { Condutividade } \\
(\mathrm{xS} / \mathrm{cm})\end{array}$} & \multirow[b]{2}{*}{$\begin{array}{l}\text { P. osmótica } \\
\text { (bar) }\end{array}$} & \multirow[b]{2}{*}{$\begin{array}{c}\text { Pressão } \\
\text { hidrostática } \\
\text { (bar) }\end{array}$} & \multirow[b]{2}{*}{$\begin{array}{c}\text { Vazão no } \\
\text { Sistema (L/min) } \\
/ \operatorname{Re}(-)\end{array}$} & \multicolumn{2}{|c|}{ *Módulo } \\
\hline & & & & & & $\begin{array}{l}\text { *Velocidade no } \\
\text { Lúmen das } \\
\text { Fibras } \\
(\mathrm{cm} / \mathrm{s}) / \operatorname{Re}(-)\end{array}$ & $\begin{array}{c}\text { *Velocidade no } \\
\text { Casco das } \\
\text { Fibras } \\
(\mathrm{cm} / \mathrm{s}) / \operatorname{Re}(-)\end{array}$ \\
\hline $\begin{array}{c}\text { Alta } \\
\text { Salinidade }\end{array}$ & $\begin{array}{c}\text { Sol. Aq. } \\
\mathrm{MgSO}_{4}(0,3 \mathrm{M})\end{array}$ & $22(\mathrm{milliS} / \mathrm{cm})$ & 8 (bar) & 1,5 & $13 / 20150$ & - & $15 / 288$ \\
\hline $\begin{array}{c}\text { Baixa } \\
\text { Salinidade }\end{array}$ & $\begin{array}{c}\mathrm{H}_{2} \mathrm{O} \\
\text { micro./dest. }\end{array}$ & $<200(\mu \mathrm{S} / \mathrm{cm})$ & $<0,1$ (bar) & 0,5 & $0,1 / 150$ & $1 / 4$ & - \\
\hline
\end{tabular}

* Considerando os módulos M1 e M2 como se fosse um módulo único de 1300 fibras $\left(1,5 \mathrm{~m}^{2}\right)$.

Nesse processo, um fluxo osmótico ocorre da corrente de baixa salinidade (LB) em direção a corrente de alta salinidade (LA). Observe-se também um fluxo oposto do soluto por difusão. A figura 6 apresenta a variação da condutividade nas linhas, assim como a variação do nível da água no tanque $\mathrm{T} 2$ ao longo do teste de osmose direta.

Como a variação de volume do tanque de baixa salinidade (T2) é diretamente relacionada com o fluxo permeado através das membranas, a derivada d $\left(\mathrm{Vol}_{\text {. }} \mathrm{H}_{2} \mathrm{O}\right) / \mathrm{d}($ tempo $)$ corresponde ao perfil da vazão permeada durante o teste. Por conseguinte, a vazão dividida pela área específica total útil das membranas acondicionadas nos permeadores M1 e M2, corresponde ao fluxo permeado. A figura 7 apresenta o perfil da vazão (Q) e do fluxo permeado (F) ao longo do teste de osmose direta. 


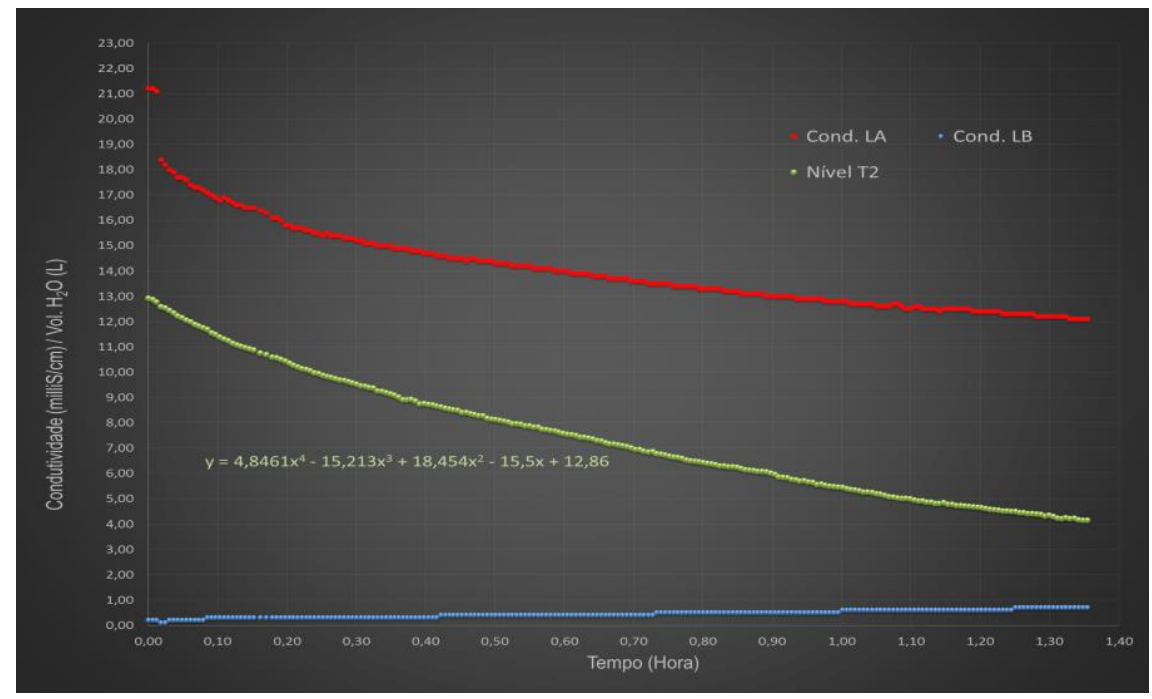

Figura 6 - Variação da condutividade das linhas de alta (LA) e baixa salinidade (LB) ao longo do teste de osmose direta (LA: $\mathrm{MgSO}_{4}$ 0,03M, $\pi$ : 8bar; LB: $\mathrm{H}_{2} \mathrm{O}$ micro./dest. $\leq 200 \mu \mathrm{S} / \mathrm{cm}, \pi$ : $\leq 0,1$ bar). Variação do nível de água no tanque T2 (Nível T2).

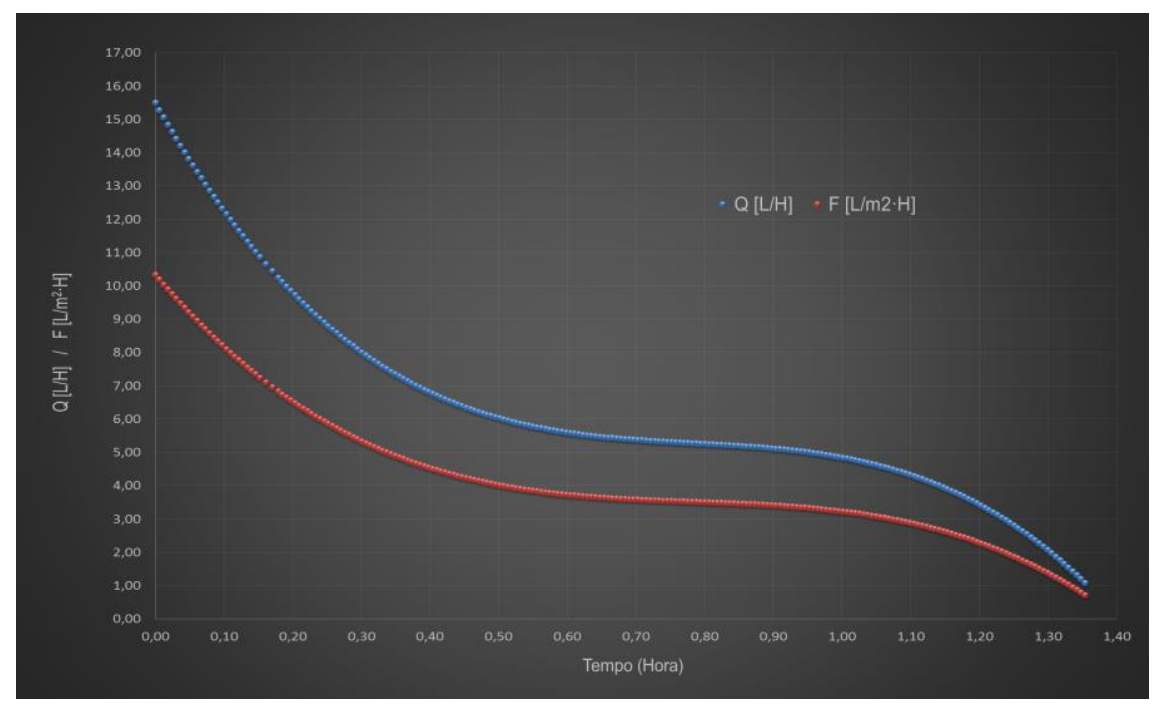

Figura 7 - Perfis da variação da vazão $(\mathrm{Q})$ e do fluxo permeado (F) ao longo do teste de osmose direta (LA: $\mathrm{MgSO}_{4}$ 0,03M, $\pi$ : 8bar; LB: $\mathrm{H}_{2} \mathrm{O}$ micro./dest. $\leq 200 \mu \mathrm{S} / \mathrm{cm}, \pi$ : $\leq 0,1$ bar).

A variação de condutividade provoca uma diminuição do gradiente de salinidade próxima as superfícies da membranas e, por conseguinte, reduz a pressão osmótica responsável pela força motriz do processo. 
A permeabilidade (B) do soluto nas membranas e a resistência ao transporte $(\mathrm{K})$ do soluto na região porosa da membrana são calculadas a partir dos resultados dos testes de osmose inversa e osmose direta.

\section{CONCLUSÕES}

O desempenho das membranas do tipo fibras ocas produzidas nesse trabalho pode ser comparado com o da membrana comercial de triacetato de celulose (CTA) da Hydration Technology Innovations, considerada como a membrana mais adequada no mercado para o processo de osmose direta (Achilli et al., 2010).

A Tabela 4 apresenta as propriedades de transporte dessas membranas. Observe-se que o desempenho da AC_2B é próximo ao da CTA, mesmo testada com menor força osmótica. A membrana produzida apresenta uma resistência ao transporte $(\mathrm{K})$ menor em relação a da CTA, o que reduz o efeito da polarização de concentração próxima a camada densa das membranas. Esse fenômeno provoca uma diminuição do gradiente de salinidade e, por conseguinte, uma diminuição da pressão osmótica.

Tabela 4 - Tabela comparativa das propriedades características de transporte da membrana do tipo fibras ocas AC_2B e da membrana comercial de CTA da Hydratation Tech. Inn. testadas com uma solução de sulfato de magnésio $\left(\mathrm{MgSO}_{4}\right)$.

\begin{tabular}{|c|c|c|c|c|c|}
\hline Membranas Ref. & $\Pi[\mathrm{bar}]$ & $\mathrm{F}_{\mathrm{w}}\left[\mathrm{L} / \mathrm{m}^{2} \cdot \mathrm{h}\right]$ & $\mathrm{F}_{\mathrm{s}}\left[\mathrm{g} / \mathrm{m}^{2} \cdot \mathrm{h}\right]$ & $\mathrm{B}[\mathrm{m} / \mathrm{s}]$ & $\mathrm{K}[\mathrm{s} / \mathrm{m}]$ \\
\hline CTA Hydra. Tech. & 14 & 4,25 & 0,9 & $\mathrm{ND}$ & $8 \cdot 10^{5}$ \\
\hline AC_2B & 8 & 3,50 & 0,7 & $3,2 \cdot 10^{-7}$ & $4 \cdot 10^{6}$ \\
\hline
\end{tabular}

No caso de uma unidade real, o sistema funcionaria sem reciclo das correntes, as quais seriam alimentadas em contínuo pelas fontes naturais, ou seja, pelo mar e por um rio de água doce, por exemplo. Dessa forma, a redução do gradiente de salinidade seria unicamente devido a polarização de concentração. Tal efeito pode ser parcialmente controlado pela intensidade do regime de escoamento das correntes.

\section{REFERÊNCIAS}

AABERG, R.J. Osmotic power: a new and powerful renewable energy source? Refocus, v. 4, pp. 48-50, 2003.

ACHILLI, A.; CHILDRESS, A. E. Selection of inorganic-based draw solutions for forward osmosis applications, Journal of Membrane Science, v. 364, pp. 233-241, 2010. 